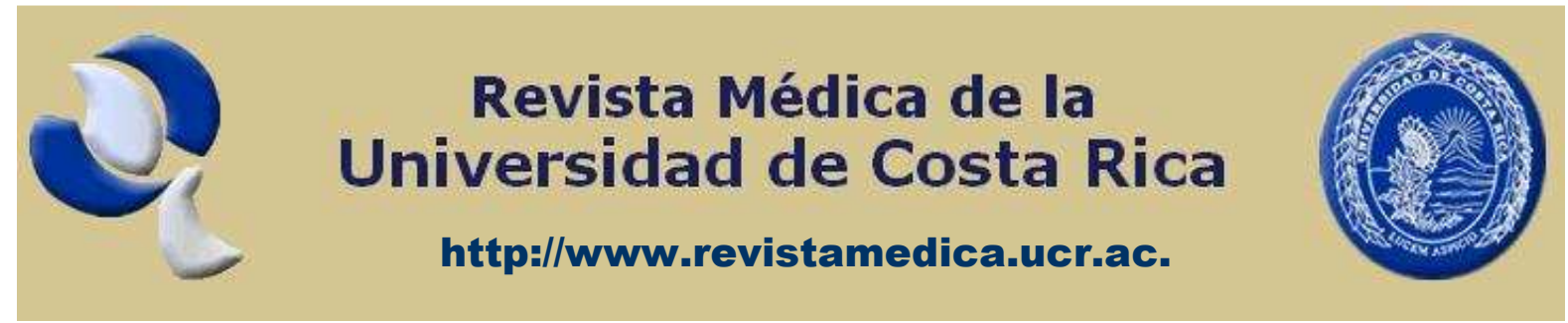

Reporte de Casos

\title{
Mutación 20210G/A del gen de la protrombina en una familia costarricense
}

\author{
Salazar Sánchez, Lisbeth ${ }^{\text {; }}$ Jiménez Cruz, Guillermo²; Chaverri Sáenz, Pilar²; \\ Ramos Alfaro, Victoria ${ }^{1}$ y Falko Holst, Herrmann ${ }^{3}$. \\ ${ }^{1}$ Centro de Investigación en Hematología y Trastornos Afines, Universidad de Costa Rica. \\ ${ }^{2}$ Servicio de Hematología Hospital México, San José-Costa Rica. \\ ${ }^{3}$ Instituto de Genética Humana, Universidad de Greifswald, Alemania.
}

Resumen: La mutación del gen de la protombina (PTG20210A), es un importante marcador asociado a un mayor riesgo de trombosis. Objetivo: se presenta el caso de una paciente con eventos trombóticos. Materiales y métodos: La paciente con historia de tromboflebitis y abortos sin factores de riesgo conocidos para ello. Se realizaron análisis de coagulación y estudios moleculares para el factor $\mathrm{V}$ Leiden (FVL), la mutación C677T de la metilentetrahidrofolato reductasa (MTHFR C677T) y de la mutación de PTG20210A. También, se analizaron tres hijos de ella. Resultados: La mutación del FVL no fue encontrada en este paciente ni en ningún miembro de su familia. La paciente es heterocigota para la MTHFR C677T y homocigota para la variante PTG20210A. Tres hijos suyos son heterocigotas para esta última mutación. Conclusiones: La variante de la PTG20210G se asociada con eventos de trombosis venosa. En el presente caso, ha sido importante este hallazgo para determinar las medidas terapéuticas de esta paciente y para tomar medidas preventivas de eventos trombóticos en los miembros de su familia que la portan.

Palabras clave: Trombofilia, tromboflebitis, trombosis venosa, protombina, mutación PTG20210A, factor V Leiden, metilentetrahidrofolato reductasa.

Recibido: Julio 2007. Aceptado: Agosto. Publicado: Septiembre 2007.

Revista electrónica publicada por la Escuela de Medicina de la Universidad de Costa Rica, 2060 San José, Costa Rica. ® All rights reserved. 
Abstract: The G20210A mutation of the prothrombin gene is an important genetic marker associated with an increased risk for thrombosis. Aim: To present a case of a Costa Rican patient with thrombotic events. Material and Methods: Case: a female with thrombophlebitis and history of miscarriages without common risk factors of thrombosis. Special Coagulation tests and DNA-marker analyses (factor V leiden, C677T metilentetrahidrofolate reductase polymorphism and PTG20210A mutation) were performed. Three sons of her were also analyzed for the DNA markers. Results: Factor $\checkmark$ Leiden mutation was not found in this patient, however she is heterozygous for MTHFRC677T and homozygous for the G20210A prothrombin mutation. Her three sons are, as expected, heterozygous for this allele. Conclusions: The PTG20210A could be considered a highly probable cause of the thrombotic events in this patient; the knowledge of the heterozygous status of her sons could also be important to prevent thrombotic events.

Keywords: Thrombophilia, thrombophlebitis, venose thrombosis, prothrombin, PTG20210A mutation, Factor V Leiden, metilentetrahidrofolate reductase,

\section{Introducción}

Uno de los trastornos vasculares más importantes es la enfermedad tromboembólica, que se caracteriza por la presencia de un trombo o émbolo en el interior de los vasos sanguíneos. Este trombo puede formarse en cualquier zona del sistema circulatorio o circular a través del torrente sanguíneo $[1,2]$. El émbolo, puede alojarse en el cerebro, pulmones, corazón o en otra área del cuerpo y causarle daño.

Las trombosis venosas se pueden dividir en trombosis de vasos superficiales y de vasos profundos. El trombo venoso se caracteriza por la oclusión total o parcial del vaso, esta constituido por fibrina y hematíes y por una participación variable de leucocitos y plaquetas; presenta un aspecto rojizo. La trombosis venosa resulta de un proceso multifactorial en el que intervienen simultáneamente diferentes condiciones, como la hipercoagulabilidad, la estasis y la Este último es una variación genética de la región 3' no codificante del gen de la PT; corresponde a una sustitución de alteración de la pared vascular. A estos tres factores se les conoce como la tríada de Virchow; la estasis sanguínea se considera la principal causa de su formación. Actualmente la trombosis venosa es la que presenta mayor impacto tanto en morbilidad como en mortalidad, entre ellas las más importantes son la trombosis venosa profunda y la embolia pulmonar [2]. Actualmente, se han definido varios factores de riesgo trombótico de origen genético que se han relacionado con la trombofilia: la mutación G1691A del factor V, denominado FV Leiden (FVL) locus 1q23, Online Mendelian Inheritance in Man (OMIM) 227400, la mutación C677T del gen que codifica para la enzima metilentetrahidrofolato reductasa (MTHFR) locus 1 p36.3, OMIN 607093 relacionada con hiperhomocisteinemia y, el polimorfismo del gen de la protrombina (PTG20210A,) locus 11p11-q12, OMIN 176930 [3,4,5]. $G$ por A en la posición 20210 (G20210A).

Revista electrónica publicada por la Escuela de Medicina de la Universidad de Costa Rica, 
Esta mutación se describió en 1996 y se asoció con la trombosis venosa, debido a que se determinó un aumento del nivel plasmático de la protombina, especialmente en los homocigotos (A/A) $[5,6]$. La elevación plasmática de la protrombina se asocia con la inhibición de la proteína $S$, cofactor de la proteína $C$ activada (PCA); también disminuye la actividad anticoagulante no dependiente de la PCA lo que aumenta el riesgo de trombosis $(6,7)$. Así, los heterocigotos de la PTG20210A (G/A), presentan un incremento de 3 veces el riesgo de sufrir un evento trombótico venoso comparado con individuos normales homocigotos $(\mathrm{G} / \mathrm{G}) \quad[6,7,8,9]$. La importancia de la mutación PT G20210A en la trombosis arterial aún no es suficientemente clara; algunos estudios sugieren que podría ser un riesgo de

\section{Materiales y Métodos}

Se trata de una mujer de 38 años de edad, con antecedentes familiares de hipertensión, enfermedades oncológicas, abortos y episodios de tromboflebitis quien es referida al CIHATA de la Universidad de Costa Rica, para un estudio de trombofilia. No presenta en su historia clínica factores de riesgo conocidos para estos eventos trombóticos.

Se le realizan pruebas de coagulación básicas y de inhibidores de coagulación, utilizando los reactivos de la casa Stago, osea el tiempo de protrombina (Neoplastine), el tiempo parcial de tromboplastina (CKPrest) y el fibrinógeno por el método de Clauss (Fibriprest) [13]. Todas se practicaron por los métodos convencionales, con las técnicas coagulométricas de la casa Stago. Los inhibidores de coagulación infarto agudo del miocardio (IAM) en fumadores jóvenes [8]. La prevalencia de este polimorfismo ha sido estudiado en varias poblaciones siendo más común en el sur (2,3\%-6,5\%) que el norte $(1,2 \%-2,6 \%)$ de Europa, y es muy rara en Asia y en individuos de ascendencia asiática [10]. En un grupo de Groenlandia no se encontró la mutación y en Costa Rica la prevalencia es del $2 \%[10,11,12]$.

En el Centro de Investigación en Hematología y Trastornos Afines (CIHATA), se han establecido y se puede ofrecer las pruebas moleculares para el FVL, MTHFR y PTG20210A con el fin de poder analizar casos de pacientes, donde se desconoce la causa de su enfermedad trombótica.

que se analizaron fueron la antitrombina III, la proteína $C$ y la proteína $S$ que se evaluaron también mediante los métodos convencionales descritos y con reactivos de la casa Stago [14,15,16].

También se le realizaron los estudios del síndrome de antifosfolípidos, o sea el anticoagulante lúpico y los anticuerpos anticardiolipinas, según lo indicado por el I Subcomité de Estandarización de la Sociedad Internacional de Hemostasia y Trombosis [17]. Pruebas de biología Molecular: Se obtuvo el ADN genómico, utilizando el método de Miller et al [18]. El factor $V$ de Leiden y el polimorfismo C677T del gen de la metilentetrahidrofolato reductasa se evaluaron según lo descrito en la literatura $[3,4]$. Para la variante PTG20210A, se utilizó el método descrito por Poor et al, [5]. 


\section{Resultados}

La paciente no presentó alteración en las pruebas básicas de coagulación; los valores de tiempo de protombina (14 segundos; control 13 segundos), tiempo de tromboplastina parcial (34.5 segundos, rango normal 25 a 35 segundos) y del fibrinógeno $335 \mathrm{mg} / \mathrm{dl}$, se encontraron dentro de los rangos normales.

La prueba de anticoagulante lúpico fue negativo y los anticuerpos anticardiolipina IgG 14 UGPL $\quad(<$ 20UGPL) y la IgM 6 UMPL (<11UMPL) fueron normales (ver tabla \# 1).

\section{Tabla \#1. Resultados de los inhibidores de la coagulación y de las pruebas moleculares realizadas a la paciente}

\begin{tabular}{|c|c|c|}
\hline $\begin{array}{l}\text { Prueba realizada } \\
\text { Inhibidores de la Coagulación }\end{array}$ & Resultado & $\begin{array}{lcc}\text { MECANISMO } & \text { EN } & \text { CASO } \\
\text { DEFICIENCIA O ANORMALIDAD }\end{array}$ \\
\hline Proteína S & $104 \%$ (normales $70-130 \%$ ) & $\begin{array}{l}\text { Falta el cofactor para la proteína C. } \\
\text { No hay inactivación del } \mathrm{Va} \text { y del } \\
\text { VIIlla. }\end{array}$ \\
\hline Antitrombina III & $114 \%$ (normales $80-120 \%$ ) & $\begin{array}{l}\text { Falla de inhibición de factores II a, } \\
\mathrm{X} \text { a otros. }\end{array}$ \\
\hline Proteína C & $126 \%$ (normales $70-130 \%$ ) & $\begin{array}{l}\text { No hay inactivación del } \mathrm{Va} \text { y del } \\
\text { VIIla }\end{array}$ \\
\hline $\begin{array}{l}\text { Marcadores } \quad \text { Moleculares } \\
\text { Analizados }\end{array}$ & & \\
\hline $\begin{array}{l}\text { Mutación del factor II } \\
\text { protrombina } \\
\text { Factor V Leiden }\end{array}$ & $\begin{array}{l}\text { Homocigota } \\
\text { No presenta mutación }\end{array}$ & $\begin{array}{l}\text { Aumento de los niveles de factor II } \\
\text { Falta de la inactivación del factor } \\
\text { Va y VIIla }\end{array}$ \\
\hline $\begin{array}{l}\text { C677T del gen de la } \\
\text { Metilentetrahidrofolato } \\
\text { reductasa }\end{array}$ & Heterocigota & $\begin{array}{l}\text { Niveles aumentados de } \\
\text { homocisteína. La homocisteína se } \\
\text { asocia con citotoxicidad endotelial } \\
\text { y alteración } \\
\text { los mecanismos hemostáticos }\end{array}$ \\
\hline
\end{tabular}

Debido a los resultados anteriores se procedió a realizar el estudio molecular

\section{Discusión}

La tromboflebitis superficial que afecta las venas safena, es más frecuente que la TVP y se trata con analgesia, sostén elástico y reposo. Las venas afectadas se palpan sensibles, calientes, eritematosas, y rara vez embolizan [19]. de la PTG20210A en el núcleo familiar de la paciente.

Revista electrónica publicada por la Escuela de Medicina de la Universidad de Costa Rica, 2060 San José, Costa Rica. ® All rights reserved.

En el presente caso, una vez descartadas los causas de trombosis adquirida (por ejemplo vasculitis $y$ anticoagulante lúpico) y haber presentado abortos, se consideró importante realizar los estudios 
diagnósticos de trombofilia. Así se incluyeron el análisis de los marcadores trombóticos, el FVL, el MTHFRC677T y la PTG20210A, en conjunto con un panel de hipercoagulabilidad hereditaria. En el presente caso, con valor significativo es el hallazgo de la PTG20210A asociado con su patología ya que la forma heterocigota de la MTHFRC677T, no parece jugar un rol directo sobre los problemas trombóticos, y aún está en discusión el papel del homocigoto [20].

El diagnóstico de una enfermedad trombótica hereditaria puede ser realizado en aproximadamente un 50\% de todos los casos de trombosis venosa $[1,2]$.

Es importante señalar que la mutación de PTG20210A, es el segundo marcador genético asociado con la trombosis luego del $\mathrm{F} V$ Leiden [1, 2, 21]. Está descrito que la presencia de la mutación se asocia con un aumento en el nivel plasmático de la protombina (FII) de la coagulación. De esta forma, se ha descrito que los individuos heterocigotos tienen de 3 a 11 veces mayor riesgo de trombosis venosa, presentan valores iguales o superiores al $30 \%$ de FII plasmático, y en el homocigoto se reporta hasta un $70 \%$ más de este factor de la coagulación [21,22]. Esta condición predispone, y se asocia, con eventos trombóticos, y puede complicarse con las alteraciones en la función de los inhibidores de la

\section{Conclusión:}

Debido a que actualmente existen distintas opiniones que dependen de la relación entre los beneficios de conocer el "status" de individuos asintomáticos comparado con sus inconvenientes $[22,23]$. El beneficio de conocer la coagulación indicadas anteriormente. En este caso, el poder determinar la presencia de esta mutación, permitió elaborar una terapia definida y evitar los factores de riesgo conocidos de trombosis para prevenir el evento trombótico. Dentro de las medidas que se tomaron en este caso fue la anticoagulación oral (warfarina) y la consulta regular para evaluar y seguir la respuesta al tratamiento.

Recomendaciones sobre el manejo terapéutico de los heterocigotos y homocigotos de la mutación PTG20210A [20,22, 23]:

-los individuos que portan la forma homocigoto con o sin historia de trombosis reciben terapia preventiva durante situaciones de riesgo y terapia anticoagulante prolongada luego de un evento trombótico.

-en el caso de los individuos heterocigotos con una historia de trombosis serán tratados del mismo modo que aquellos pacientes con similares eventos clínicos debidos a deficiencias en las proteínas $\mathrm{C}, \mathrm{S}$ o antitrombina III.

-si el individuo es un heterocigoto sin historia personal o familiar de trombosis deben recibir terapia anticoagulante profiláctica en situaciones que pueden provocar la trombosis.

presencia de la forma heterocigoto de la PTG20210A, es el tratar de prevenir la enfermedad tromboembólica 0 de tratarla tempranamente. Dentro de las desventajas se incluyen la angustia sufrida por el paciente que se sabe

Revista electrónica publicada por la Escuela de Medicina de la Universidad de Costa Rica, 2060 San José, Costa Rica. ® All rights reserved. 
portador de un defecto genético, pero que no sufre de ningún síntoma, además están los riesgos asociados con la prevención del tratamiento. Algunos autores afirman que si bien el tratamiento con cumarínicos de por vida no es conveniente, no hay dudas de los beneficios de la terapia anticoagulante en el momento de enfrentar situaciones de riesgo como la cirugía [22,23]. Sin duda en el caso de las portadoras femeninas, en edad fértil, es importante el considerar y evaluar los riesgos al respecto de los anticonceptivos orales,

\section{Agradecimientos:}

Al apoyo del Instituto de Genética Humana de la Universidad Greifswald, Ernst-Moritz-Arnd, al Servicio Alemán de Intercambio Académico (DAAD) y a la

\section{Bibliografía}

1. López JA, Kearon C. Lee YA. Deep Venous Thrombosis. Hematology (Am Soc Hematol Educ Program). 2004;(1):439-562.

2. Press $R$ and DeLoughery $T$. Thrombotic Risk Assessment. Clinical Laboratory News 2000;812.

3. Bertina RM, Koeleman BC, Koster $\mathrm{T}$, Rosendaal RF, Dirven RJ, de Ronde $H$, van der Velden PA, Reitsma PH. Mutation in blood coagulation factor $\mathrm{V}$ associated with resistance to activated protein C. Nature 1994; 369: 64-67.

4. Frosst $P$, Blom $\mathrm{HJ}$, Milos $R$, Goyette $P$, Sheppard CA, Matthews RG, Boers GJ, den Heijer M, Kluijmans LAJ, van den Heuvel LP, Rozen R. A candidate genetic risk factor for vascular diseases: a common mutation in methylenetetrahydrofolate reductase. Nat Genet 1995; 10:111-113.

5. Poort SR, Rosendaal FR, Reitsma PH, Bertina RM. A common genetic variation in the $3^{\prime}$ untranslated region of the prothrombin gene is associated with elevated plasma prothrombin como lo han demostrado varios autores, el riesgo de sufrir trombosis en pacientes portadoras que consumen anticonceptivos, aumenta en heterocigotos y en homocigotos [23]. Finalmente, es valedero tener en cuenta que cualquier individuo que presente esta mutación, así como la del FVL, debe ser asesorado sobre las situaciones de riesgo secundario y se les debe ofrecer a sus familiares directos la posibilidad de realizarse el análisis.

Vicerrectoría de Investigación, Universidad de Costa Rica (proyecto No. 807-A2-313).

levels and an increase in venous thrombosis. Blood 1996; 88: 3698-3703.

6. González Ordóñez A, Medina Rodríguez J, Fernández Alvarez C y cols. Mutación 20210 A del gen de la protrombina y tromboembolismo venoso. Sangre 1999;44:13-18.

7. Batlle J y López Fernández M. Importancia del estudio del factor $\mathrm{V}$ Leiden y del alelo 20210 A del gen de la protrombina en la enfermedad tromboembólica. Sangre 1999; 44:3-6.

8. Arruda VR, et al. Prevalence of the prothrombin gene variant (nt20210A) in venous thrombosis and arterial disease. Thromb Haemost 1997;78:1430-1433.

9. Salazar -Sanchez L, Leon MP, Cartin M, Schuster G, Wulff K, Schroder W, Jimenez-Arce G, Chacon R, Herrmann FH. The FXIIIVal34Leu, common and risk factors of venous thrombosis in early middle-age Costa Rican patients. Cell Biochem Funct. Dec 292006.

Revista electrónica publicada por la Escuela de Medicina de la Universidad de Costa Rica, 2060 San José, Costa Rica. ® All rights reserved. 
10. Rosendaal FR, Doggen CJ, Zivelin A, Arruda VR, Aiach M, Siscovick DS et al. Geographic distribution of the $20210 \mathrm{G}$ to A prothrombin variant. Thromb Haemost 1998; 79: 706-708.

11. Hessner MJ, Luhm RA, Pearson SL, Endean DJ, Friedman KD, Montgomery RR. Prevalence of prothrombin G20210A, factor V G1691A (Leiden), and methylenetetrahydrofolate reductase (MTHFR) C677T in seven different populations determined by multiplex allelespecific PCR. Thromb Haemost 1999; 81: 733738.

12. Herrmann FH, Salazar-Sanchez L, Schröder W, Grimm R, Schuster G, Jimenez-Arce G, Chavez M, Singh JR. Prevalence of molecular risk factors, FVLeiden, FVHR2, FIIG21210A and MTHFR $677 \mathrm{C}>\mathrm{T}$ in different populations and ethnic groups of Germany, Costa Rica and India. Intl J. Hum Genet 2001; 1:33-39.

13. Clauss A. Gerinnungsphysiologische Schnellmethode zur Bestimmung des Fibrinogen. Acta Haematol 1957; 17: 237-246.

14. Borg JL, Duriez D, Thirion C, Nicham F, Perry DJ, Carrell RW. Improvement in the identification of antithrombin deficient patients. XVIth congress of the international Society on Thrombosis and Haemostasis. Thromb Haemost 1997; 433(Suppl) 1768.

15. Guglielmone HA, Vides MA. A novel functional assay of protein $C$ in human plasma and its comparison with amidolytic and anticoagulant assays. Thromb Haemost 1992; 67: $46-49$

16. Bertina RM. Hereditary protein S deficiency. Haemostasis 1985; 15:241-246.
17. Brandt JT, Triplett DA, Alving B, Scharrer I. Criteria for the diagnosis of lupus anticoagulants: an update. On behalf of the Subcommittee on Lupus Anticoagulant/Antiphospholipid antibody of the scientific and standardization committee of the ISTH. Thromb Haemost 1995; 74: 11851190.

18. Miller M, Dykes DD, Polesky HF. A simple salting out procedure for extracting DNA from human nucleated cells. Nucleic Acids Res 1988; 16: 121.

19. Ginsberg JS, Greer I, Hirsh J. Use of antithrombotic agents during pregnancy. Chest Supplement 2001; 119(1):122S-131S.

20. Robertorye TS, Rodgers GM Update on selected inherited venous thrombotic disorders.r Am J Hum Biol. 2004;16(1):82-86.

21. Frances F, Portoles $O$, Gabriel F, et al. Comparación de las frecuencias de los alelos factor $V$ Leiden (G1691A) y protrombinaG20210A entre pacientes con trombosis venosa profunda y población general mediterránea española. Rev. méd. Chile 2006;134:1 :13-20.

22. McGlennen RC, et al. Clinical and laboratory management of the prothrombin G20210A mutation. Arch Pathol Lab Med 2002;126:13191325.

23. CAP Consensus Conference XXXVI: Diagnostic issues in thrombophilia: Introduction and general considerations. Arch Pathol Lab Med 2002;126:1277-1280.

\section{Correspondencia:}

\section{Lizbeth Salazar Sánchez}

Centro de Investigación en Hematología y Trastornos Afines, Universidad de Costa Rica.

lizbeth.salazar@gmail.com

Revista electrónica publicada por la Escuela de Medicina de la Universidad de Costa Rica, 